\title{
Comparison between transperineal ultrasound and digital detection of levator ani trauma. Can we improve the odds?
}

Jennifer A. Kruger,

Hans Peter Dietz,

Stephanie C. Budgett,

Chantale L. Dumoulin

First published: 22 February 2013

Conflict of interest: none.

Mickey Karram led the peer-review process as the Associate Editor responsible for the paper.

This study was undertaken at the Physiotherapy Laboratory at the Montreal Geriatric Institute Research Centre for Pelvic floor muscle function in aging women.

\begin{abstract}
Aims

To investigate the predictive ability of four digital assessment parameters to detect levator ani (LA) muscle defects (avulsion injury) and compare these to transperineal tomographic ultrasound images.

Methods

This was an observational study imbedded in a larger quasi-experimental cohort study for women with urinary incontinence. Seventy-two women, $\geq 60$ years who had attended or were going to attend physiotherapy for treatment of urinary incontinence, were included in the study. Inclusion criteria from the parent study were symptoms of stress, urge or both types of urinary incontinence. The predictive ability of the following digital parameters: direct palpation of a discontinuity of the LA muscle from insertion on the pubic ramus; palpation of the distance between the muscle insertion sites; palpation of LA strength; palpation of LA tone, were analyzed against findings from tomographic transperineal ultrasound images. Correlation between methods was measured using Cohen's kappa for each of the individual parameters.
\end{abstract}

Results

Seventeen women (24\%) presented with a complete or partial avulsion of the puborectalis muscle as diagnosed with tomographic ultrasound imaging. Nine women (13\%) had complete avulsions, one of which was bilateral. The predictive ability of the digital assessment parameters varied from poor $(\mathrm{k}=0.187,95 \%$ CI [0.02-0.36]) to moderate $(\mathrm{k}=0.569,95 \%$ CI [0.31-0.83]). The new parameter of "width between insertion sites' performed best.

Conclusions

Adding the parameter of "width between insertion sites" appears to enhance our ability to detect avulsion of the levator ani (LA) muscle by digital examination however it does not distinguish between unilateral or bilateral avulsion.

\section{INTRODUCTION}

Childbirth related trauma to the pelvic floor muscle, or levator ani (LA) muscle, has been shown to occur in $10-30 \%$ of women delivering vaginally.[1,2] Injury to the muscle, typically at the bone/muscle interface on the inferior pubic ramus, has been confirmed using ultrasound and magnetic resonance imaging.[3, 4$]$ Pelvic floor muscle injury is significantly associated with pelvic floor muscle dysfunction; which usually presents as either pelvic organ prolapse (POP) or urinary incontinence. $[\underline{5,6}]$

Anterior pelvic floor muscle function is traditionally determined by digitally palpating the LA muscles. It forms the basis of any vaginal examination and assessment before intervention for POP or urinary incontinence. Nonetheless, previous studies that have used traditional methods of assessment, have found 
poor individual correlation with imaging modalities in the detection of a LA avulsion injury.[7-9] In particular, assessing passive strength (tone), LA active strength using Oxford grading, and feeling for a palpable detachment of the muscle from the inferior pubic ramus found only poor to moderate correlation with transperineal ultrasound images and MR imaging.[8, 9] This is unfortunate, as digital palpation remains the first line of assessment for LA function without any additional costs, be they equipment, expertise or time.

In spite of this, we hypothesized that it may be possible to improve detection by combining the traditional assessment parameters to a measurement similar to that usually done to assess the width of a rectus abdominus diastasis recti.[10] In this case the distance between the puborectalis muscle insertion sites on the pubis (width between insertion sites). Previously, good inter-rater evaluator repeatability for the transverse diameter of the vaginal opening at mid-hiatus, has been reported (ICC 0.6).[11] The rationale for using this additional parameter is that if this distance were large then there would be an increased probability of the muscle being detached from the pubic bone, at least on one side.

The objective of this study was therefore, to assess the predictive value of the following parameters used to detect the presence of an avulsion injury, individually and in combination, in a cohort of predominately urinary stress incontinent elderly women: (1) direct palpation of a discontinuity of muscle where it attaches to the pubic ramus (absence of muscle), (2) palpation of the distance between the puborectalis muscle insertion sites (width between insertion sites), (3) palpation of LA strength, (4) palpation of LA tone. All digital assessment parameters would be performed subsequent to voluntary activation of the muscle. These results would then be compared to the accepted standard results obtained from tomographic ultrasound images of the LA complex.

\section{MATERIALS AND METHODS}

\section{Subjects}

We recruited 72 women, aged over 60 years, from a larger parent study that characterized and compared the morphology and function of the female pelvic floor in continent and incontinent community dwelling, women. All participants had attended or were going to attend physiotherapy for treatment of urinary incontinence. The type of urinary incontinence was assessed using the Urinary Distress Inventory (UDI) and the International Consultation on incontinence questionnaire (ICIQ Short Form).[12]

Ethical approval was granted by the comité mixte d'éthique de la recherche of Regroupement Neuroimagerie/Québec (CMER-RNQ), IUGM.

Palpation Protocol

Participants were examined supine, with the knees comfortably flexed after voiding by an experienced pelvic floor muscle physiotherapist (CD). Initial observation of the introitus determined if the participant was able to perform a correct contraction, evidenced by an inward movement of the perineum. If not, time was taken to teach a correct contraction. All participants were then instructed to perform a series of voluntary contractions as proposed by the Laycock's PERFECT scheme (2001).[13] We felt the predictive value of palpation could be improved by trying to increase muscle definition prior to assessment. It is well recognized that acute bouts of intense concentric exercise increases intracellular fluids evidenced by using MR imaging.[14, 15] As the LA muscles act concentrically during exercise, increasing intracellular fluid may make them easier to palpate.

Three contractile tasks were completed by the subject: Endurance-length of time the muscle can maximally contract (up to $10 \mathrm{sec}$ ); Repetition - number of repetitions (up to 10) which the muscles can be maximally contracted (up to 10 repetitions); and Fast-number of contractions that can be performed rapidly (up to 10).

Digital assessment of LA integrity was then conducted and included the following: (1) feeling for continuity of the puborectalis muscle at its insertion sites on either side of the pubic bone. A positive result (avulsion) was recorded if it was possible to feel a discontinuity of the muscle with the bone, or absence of muscle on each side, (2) determining the distance between the insertion points of the puborectalis muscle 
measured using finger widths, that is, three fingers equate to approximately $4 \mathrm{~cm}$ (as with diastasis recti), which gave one measure (Fig. 1), (3) LA strength using previously validated modified Oxford grading scale $(0-5),[16]$ side dependent, and (4) grading of resting tone of the LA muscle using a verified scale, also side dependent.[17] A modified template from a previous publication[] was used to record the data from the palpation (Fig. 2). The physiotherapist performing the digital LA assessment was blinded to the ultrasound results as well as parity and mode of delivery of the participant.

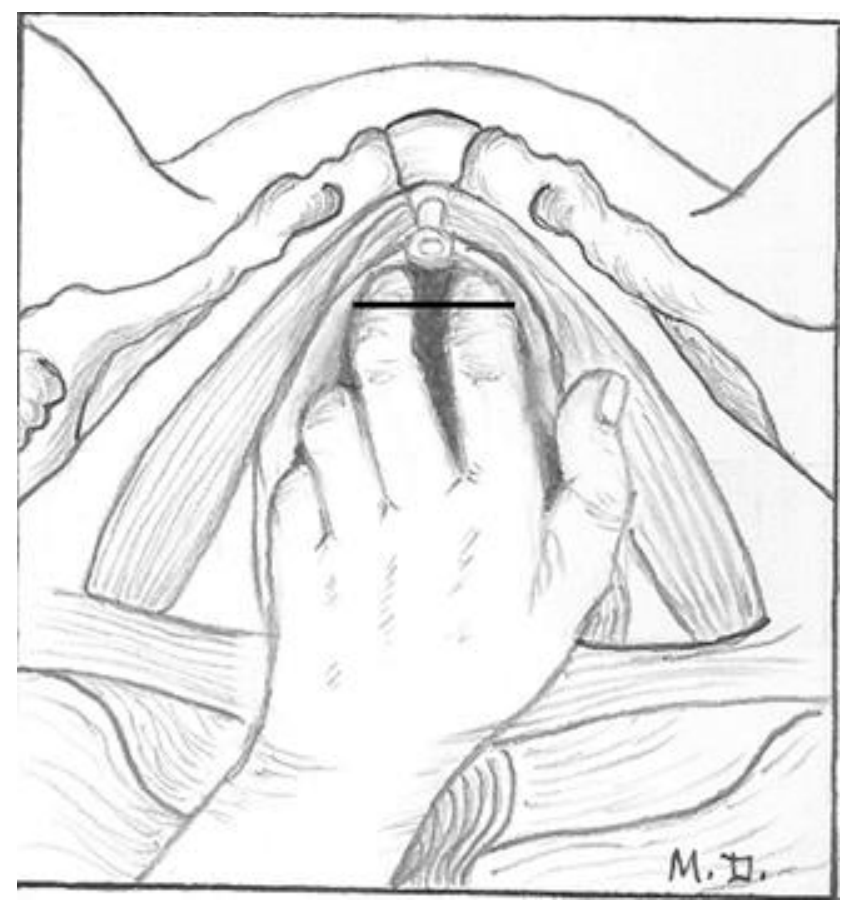

Figure 1.

Illustration indicating where the fingers are placed in the vagina to determine the new parameter "width between insertion sites". Solid line indicates where the measurement is made. 


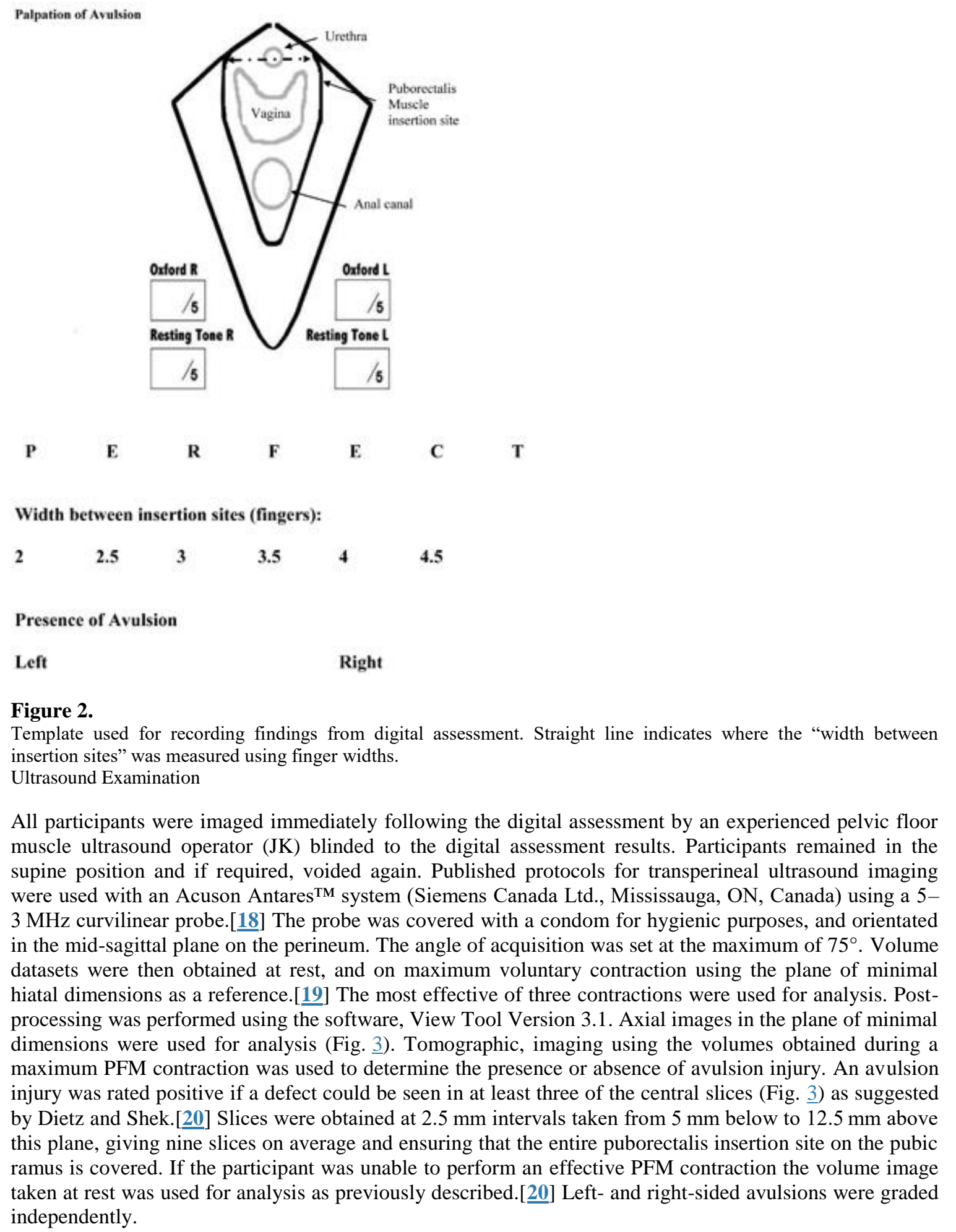




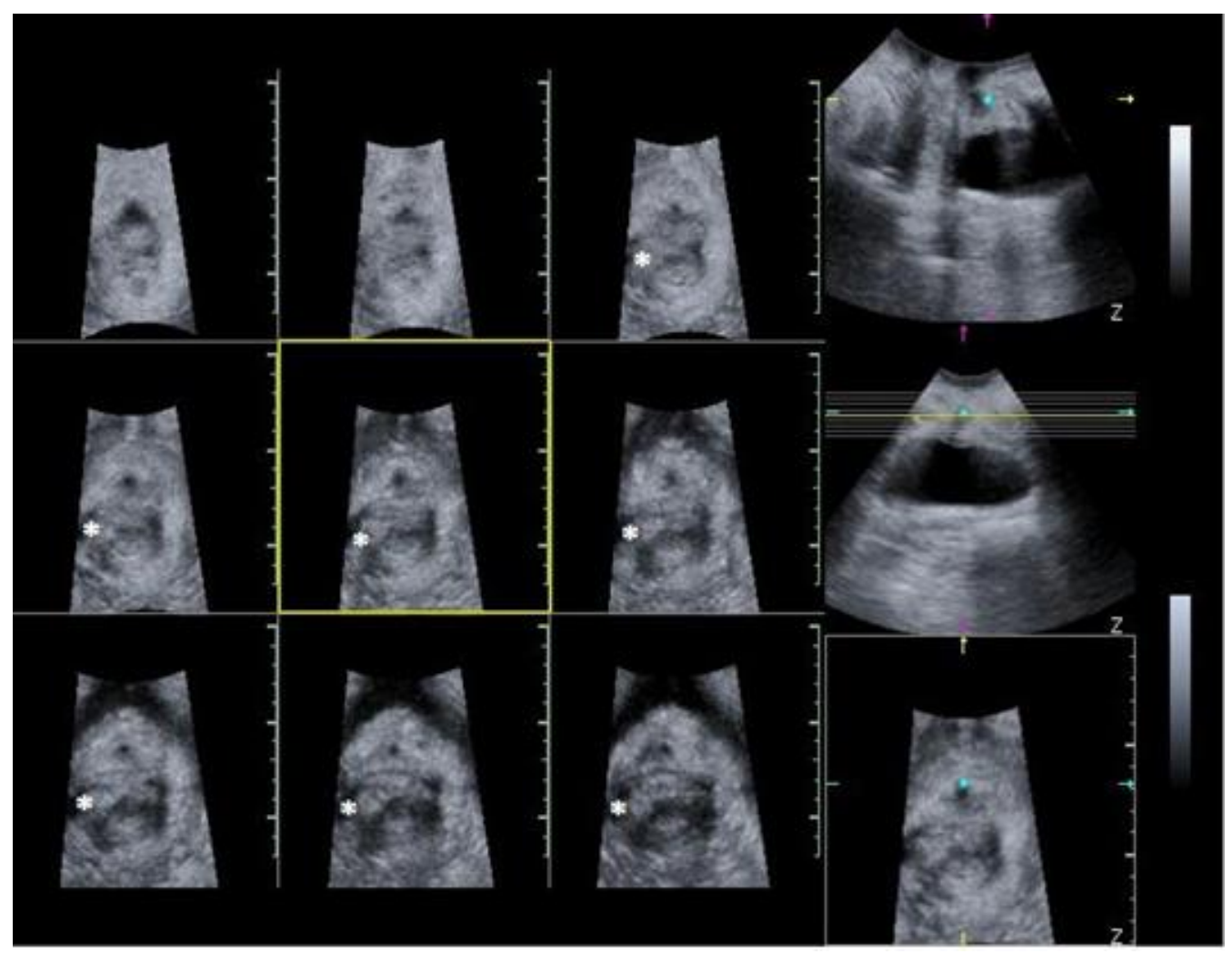

Figure 3.

Tomographic ultrasound showing right sided defect, indicated by the asterisk at the insertion site of puborectalis in all seven slices (slice interval $2.5 \mathrm{~mm}$ ).

All ultrasound images were verified remotely by an expert in ultrasound imaging of the pelvic floor muscles using tomographic ultrasound (PD). When there was disagreement in the analysis, the images were reassessed but the opinion of the expert (PD) was used in the final analysis. This had the added advantage of eliminating any potential bias on the part of the ultrasound operator JK.

Statistical Analysis

Statistical analysis was undertaken using SPSS V 18 (SPSS, Chicago, IL). Positive or negative findings for each of the maneuvers used for palpation were tested against the ultrasound findings. Overall agreement between the methods and ultrasound evaluators was assessed using Cohen's kappa. Relative strengths of agreement were rated in accordance with the scale developed by Landis and Koch.[21] If feasible, a regression analysis using the digital parameters to determine a predictive model was planned.

\section{RESULTS}

Of the 72 participants, 2 had invalid ultrasound images and 2 others were unable to be assessed digitally, one due to pain and one due to the presence of a perineal fissure. Thus 68 datasets were available for analysis, with 136-paired assessments of a right or left defect. Fifty women were parous. None of the participants who had had only a caesarean section or were nulliparous showed any evidence of avulsion on ultrasound imaging. Further demographic characteristics are shown in Table $\underline{I}$. 
Table I. Demographic Characteristics of Population

Characteristic

Age (years) mean $\pm \mathrm{SD}$, range

BMI $\left(\mathrm{kg} / \mathrm{m}^{2}\right)$ mean, range

Parity (median, range)

Vaginal births $(\mathrm{n}=50)$ (median, range)

Caesarean section $(n=4)$
69, $6.360-87$

$25.9,4.6,14.4-40.2$

$1,0-11$

$1,0-11$

$0,0-1$

Seventeen of 72 women (28\%) reported stress incontinence; only 1 complained of urge incontinence and most felt they had both types of urinary incontinence (54/72). ICIQ scores ranged from 0 to 20 (mean $9.06 \pm 4.775)$. Clinical assessment of prolapse detected a cystocele stage 2 or above in 35/72 $(49.3 \%)$ of women, 11 women were found to have >stage 2 rectocele, and $4(6 \%)$ had a uterine prolapse $\geq$ stage 2 .

Seventeen women (24\%) presented with either a partial or complete avulsion of the puborectalis muscle on either side as diagnosed with tomographic ultrasound. Correlation between digital examination and ultrasound images was carried out using complete avulsions only as partial avulsion is extremely difficult to quantify digitally. Ten complete avulsions, of which 1 was bilateral (14\%), were diagnosed on ultrasound. As regards analysis of ultrasound volumes, there was agreement between the two assessors in $88 \%$ of cases $(120 / 136), \mathrm{k}=0.573,95 \%$ confidence interval $0.32-0.82$.

Palpation for continuity of the muscle at its insertion site agreed moderately well with ultrasound diagnosis of avulsion, $\mathrm{k}=0.408$. Palpating the distance between the two insertion sites at the level of the pubis and using a cut off of $\geq 3.5$ fingers was able to predict $78 \%$ of complete avulsions $(\mathrm{k}=0.569)$. An intact muscle palpated between two and three fingers. The grading of LA strength using an Oxford grading $\leq 3$, and grading of resting tone $\leq 2$, was less predictive. Agreement between palpation and ultrasound findings is shown in Table II. As no avulsions were detected in those who had not given birth vaginally, a sub analysis on this group is presented as a comparison in Table III. A regression analysis to determine a predictive model for which combination of parameters would best predict avulsion was not possible due to the small number of avulsions present in our population. 
Table II. Prediction of Complete Avulsion Injury Using Digital Assessment of the Levator Ani Muscle, Compared to Tomographic Ultrasound Imaging Diagnosis of Avulsion

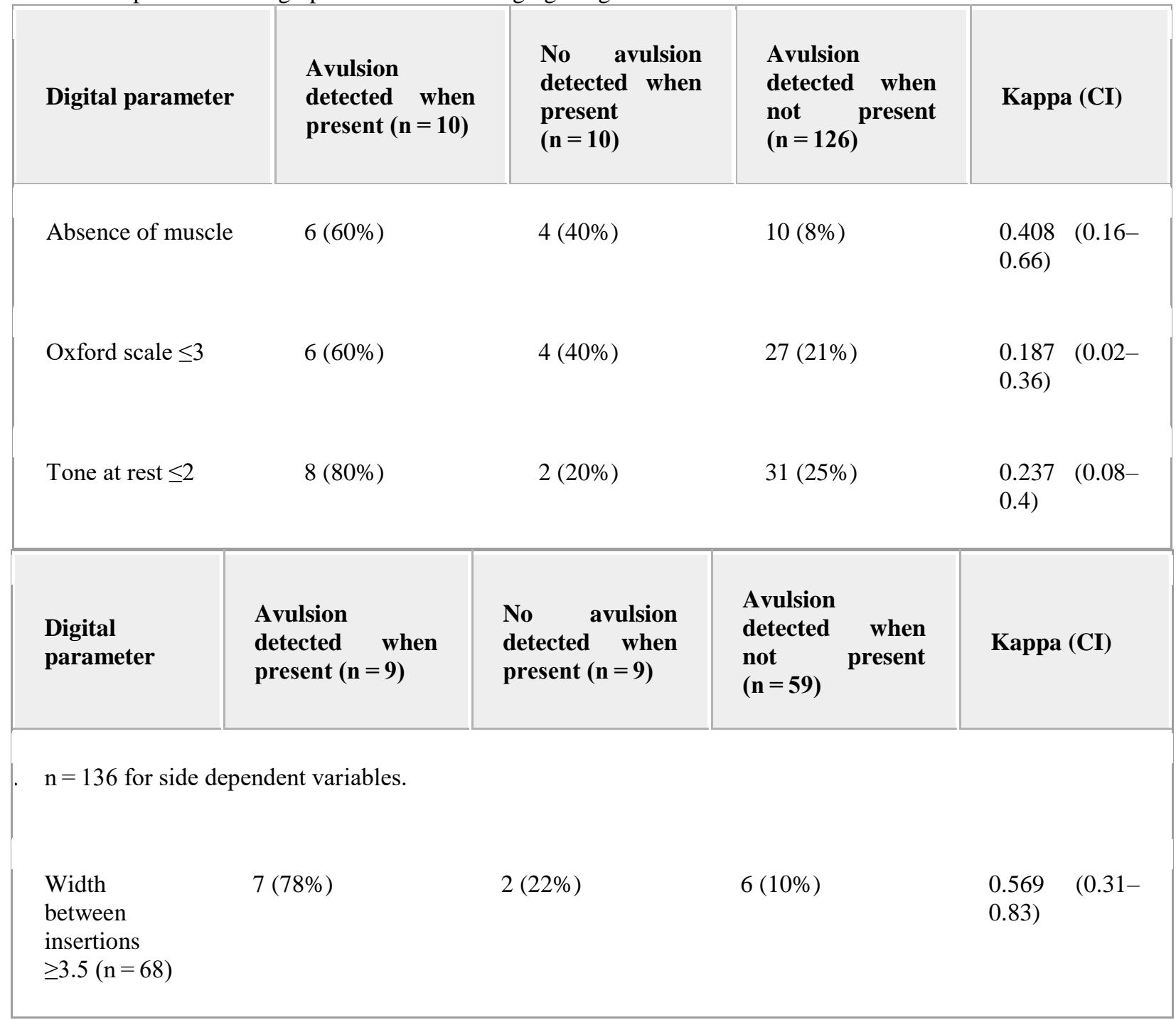

Table III. Comparison of Prediction of Avulsion Between the Total Population ( $\mathrm{n}=136$ for Side Dependent Variables and $n=68$ Non-Side Dependent) and Parous Women ( $\mathrm{n}=100$ for Side Dependent Variables, $\mathrm{n}=50$ for Non-Side Dependent)

\begin{tabular}{|l|l|l|}
\hline Digital parameter & $\begin{array}{l}\text { Total cohort }(\mathbf{n}=\mathbf{1 3 6}), \text { kappa } \\
{[\mathbf{9 5 \%} \mathbf{C I}]}\end{array}$ & $\begin{array}{l}\text { Parous women }(\mathbf{n}=\mathbf{1 0 0}), \mathbf{k a p p a} \\
{[\mathbf{9 5 \%} \mathbf{C I}]}\end{array}$ \\
\hline Absence of muscle & $0.408[0.16-0.66]$ & $0.409[0.15-0.67]$ \\
\hline Oxford scale $\leq 3$ & $0.187[0.02-0.36]$ & $0.187[0.00-0.38]$ \\
\hline Tone at rest $\leq 2$ & $0.237[0.08-0.40]$ & $0.208[0.05-0.37]$ \\
\hline
\end{tabular}


CI, confidence interval.

Width between insertion sites $\geq 3.5 \quad 0.569[0.31-0.83] \quad 0.538[0.26-0.81]$

\section{DISCUSSION}

We undertook this study to determine if the use an additional digital assessment parameter "width between insertion sites" and using voluntary activation of the muscles prior to digital assessment, would improve our ability to detect an avulsion injury. We have shown a moderate improvement, compared to other studies $(\mathrm{k}=0.569)$. Previous studies using tomographic ultrasound, and previously defined digital assessment parameters, found poor $(\mathrm{k}=0.098)$ to moderate $(\mathrm{k}=0.495)$ correlation. $[7,8]$ Using only the digital parameter of feeling for "absence of muscle" the correlation between methods was similar to that found by Dietz and Shek,[] $\mathrm{k}=0.495$, compared to $\mathrm{k}=0.408$. Kearney et al.[2] compared digital assessment of "muscle bulk" of the pubovisceral portion of the LA muscle to MR imaging, again with moderate agreement $(\mathrm{k}=0.444)$. Although these findings, including our own, demonstrate only moderate success in predicting LA defects with our fingers, it is highly likely that this could be improved with practice. While the physiotherapist was well experienced in assessing PFM function, the ability to detect a defect improved as all final five avulsions were detected digitally. This is in keeping with the previous studies that have also demonstrated a learning effect.[8,9] Moreover, the previous studies took place in tertiary urogynecology units where the proportion of women presenting with POP, and avulsion injury was higher than in our population ( $20 \%$ as compared to our $14 \%$ ). A sub-analysis of parous women did not show any significant improvement in the predictive performance of the digital parameters, despite all the avulsions being present in this group. Women with SUI have not shown the same incidence of muscle defects as those suffering primarily from POP.[22] Despite this, the predictive ability of the parameter used in this study "width between insertion sites" performed better than those previously recorded, suggesting that had this study been conducted in a population where POP was more prevalent, this parameter may perform even better.

It seems intuitive that "using our fingers" to determine injury should be a priority, particularly when the injury has been shown to be a strong risk factor for prolapse.[23, 24] Palpating discontinuity of the muscle with the bone, or absence of muscle bulk, is a relatively new addition to the routine assessment of PFM function. Nonetheless, these new techniques are not difficult to learn, as evidenced by the improved detection of defects toward the end of the study by the physiotherapist who was a novice to begin with.

Pelvic floor muscle physiotherapy is regarded as the first line treatment for urinary incontinence, and more recently Level A evidence suggests it is effective for some women with POP.[25, 26] Establishing a method that can not only accurately assess muscle function, but can also determine the presence of injury may have implications for the treatment of muscle dysfunction.

Limitations to the Study

The overall avulsion rate in this group of predominantly mixed incontinent women was rather low (14\%). Only nine women presented with a complete avulsion, as diagnosed by ultrasound imaging. The ability therefore to vigorously test the additional parameter "width between insertion sites" to assist in predicting damage, was limited. Furthermore, as this parameter is unable to distinguish between a unilateral and bilateral avulsion it should be used to enhance direct palpation of a discontinuity of muscle where it attaches to the pubic ramus.

Digital assessment of the LA muscle remains a critical component of the general assessment of the pelvic floor function. Thus, continuing to improve our ability to "feel" for muscle injury is important. 
Development of a model to determine which combination of parameters best predict avulsion injury is likely to be more successful in a population with a higher prevalence of POP.

\section{CONCLUSION}

Palpation for continuity of the puborectalis portion of the LA has been described as a method for diagnosing avulsion of the LA muscle. Our results suggest that using the simple measure of "distance in finger widths between muscle insertion points" and asking the participant to perform a series of voluntary contractions prior to assessment, could enhance the diagnosis of avulsion. It appears that there are fewer "false positives" when using this method but the caveat is that this method alone is unable to distinguish between unilateral and bilateral avulsion. Therefore it should be used in addition to direct palpation of a discontinuity of muscle where it attaches to the pubic ramus, in an effort to improve the accuracy of findings on physical examination.

\section{REFERENCES}

1 Dietz HP, Steensma AB. The prevalence of major abnormalities of the levator ani in urogynaecological patients. BJOG 2006; 113:225-30.

2 Kearney R, Miller JM, Ashton-Miller JA, et al. Obstetric factors associated with levator ani muscle injury after vaginal birth. Obstet Gynecol 2006; 107:144-9.

3 Dietz HP. Quantification of major morphological abnormalities of the levator ani. Ultrasound Obstet Gynecol 2007; 29:329-34.

4 DeLancey JOL, Kearney R, Chou Q, et al. The appearance of levator ani muscle abnormalities in magnetic resonance images after vaginal delivery. Obstet Gynecol 2003; 101:46-53.

5 Dietz HP. The aetiology of prolapse. Int Urogynecol J Pelvic Floor Dysfunct 2008; 19:1323-9.

6 Delancey JO, Kane Low L, Miller JM, et al. Graphic integration of causal factors of pelvic floor disorders: An integrated life span model. Am J Obstet Gynecol 2008; 199:610.e1-5.

7 Dietz HP, Hyland G, Hay-Smith J. The assessment of levator trauma: A comparison between palpation and 4D pelvic floor ultrasound. Neurourol Urodyn 2006; 25:424-7.

8 Dietz HP, Shek C. Validity and reproducibility of the digital detection of levator trauma. Int Urogynecol J Pelvic Floor Dysfunct 2008; 19:1097-101.

9 Kearney R, Miller JM, Delancey JO. Interrater reliability and physical examination of the pubovisceral portion of the levator ani muscle, validity comparisons using MR imaging. Neurourol Urodyn 2006; 25:504.

10 Wilder E, editor. Obstetric and Gynecologic physical therapy. New York: Churchill Livingston; 1988.

11 Boyles SH, Edwards SR, Gregory WT, et al. Validating a clinical measure of levator hiatus size. Am J Obstet Gynecol 2007; 196:174.e1-4.

12 Madill S, Tang A, Pontbriand-Drolet S, et al. Comparison of two methods for measuring the pubococcygeal line from sagittal-plane magnetic resonance imaging. Neurourol Urodyn 2011; 30:1613-9.

13 Laycock J, Jerwood D. Pelvic floor muscle assessment: The PERFECT Scheme. Physiotherapy 2001; 87:631-42. 
14 Fleckenstein J, Canby R, Parkey R, et al. Acute effects of exercise on MR imaging of skeletal muscle in normal volunteers. Am J Roentgenol 1988; 151:231-7.

15 Shellock F, Fukunaga T, Mink J, et al. Acute effects of exercise on MR imaging of skeletal muscle: Concentric vs eccentric actions. Am J Roentgenol 1991; 156:765-8.

16 Bo K, Finckenhagen HB. Vaginal palpation of pelvic floor muscle strength: Inter-test reproducibility and comparison between palpation and vaginal squeeze pressure. Acta Obstet Gynecol Scand 2001;

80:883-7.

17 Dietz H, Shek K. The quantification of levator muscle resting tone by digital assessment. Int Urogynecol J 2008; 19:1489-93.

18 Dietz HP. Pelvic floor ultrasound: A review. Am J Obstet Gynecol 2010; 202:321-34.

19 Dietz HP, Shek C, Clarke B. Biometry of the pubovisceral muscle and levator hiatus by threedimensional pelvic floor ultrasound. Ultrasound Obstet Gynecol 2005; 25:580-5.

20 Dietz HP, Shek KL. Tomographic ultrasound imaging of the pelvic floor: Which levels matter most? Ultrasound Obstet Gynecol 2009; 33:698-703.

21 Landis JR, Koch GG. The measurement of observer agreement for categorical data. Biometrics 1977; 33:159-74.

22 Dietz HP, Kirby A, Shek KL, et al. Does avulsion of the puborectalis muscle affect bladder function? Int Urogynecol J Pelvic Floor Dysfunct 2009; 20:967-72.

23 Dietz HP. The role of two- and three-dimensional dynamic ultrasonography in pelvic organ prolapse. $\mathrm{J}$ Minim Invasive Gynecol 2010; 17:282-94.

24 Morgan DM, Cardoza P, Guire K, et al. Levator ani defect status and lower urinary tract symptoms in women with pelvic organ prolapse Int Urogynecol J 2010; 21:47-52.

25 Hagen S, Stark D, Glazener C, et al. A Multicentre randomised controlled trial of a pelvic floor muscle training intervention for women with pelvic organ prolapse. Neurourol Urodyn 2011; 30:983-4.

26 Braekken IH, Majida M, Engh ME, et al. Can pelvic floor muscle training reverse pelvic organ prolapse and reduce prolapse symptoms? An assessor-blinded, randomized, controlled trial. Am J Obstet Gynecol 2010; 203:170.e1-7.

This is the accepted version of the following article: Kruger JA, Dietz HP, Budgett SC, Dumoulin C. (2013) Comparison between transperineal ultrasound and digital dectection of levator ani trauma. Can we improve the odds? Neurourology and Urodynamics; 33(3):307-311., which has been published in final form at http://onlinelibrary.wiley.com/doi/10.1002/nau.22386/epdf 